\title{
A Review of Image Denoising and Segmentation Methods Based on Medical Images
}

\author{
Sreedhar Kollem, Katta Rama Linga Reddy, and Duggirala Srinivasa Rao
}

\begin{abstract}
Image denoising and segmentation are required to use in digital image processing. For researchers' point of view, still, these two methods are challenging task in medical images. At present, image denoising and segmentation take part in real-world applications such as computer graphic, computer vision, satellite, and medical fields. These two methods are analyzed by using different images but mainly concentration on medical images such as computed tomography, single photon emission computed tomography, magnetic resonance imaging, positron emission tomography. Medical images can break into noise, major research has created solutions to this complication, various techniques are being proposed. Image segmentation is a widespread and active area not only for medical imaging but also for computer vision and satellite imaging. The foremost plan of image segmentation remains to segment images into different components, which are used to give more information about the medical image. Here is an overview of the different methods after a brief introduction. These methods are classified as the basis for the techniques used.
\end{abstract}

Index Terms - Image denoising, Image segmentation, performance parameters, derivative based image denoising, clustering methods.

\section{INTRODUCTION}

Digital image processing requires a computer to work with images, so it is fit aimed at image investigation, pattern recognition, together with human reflection. By means of computer knowledge, digital image processing has several applications over different industries such as face recognition, medical imaging, computer vision, remote sensing, and medical sciences.

Medical images such as computed tomography, single photon emission computed tomography, magnetic resonance imaging, positron emission tomography contains rich information, both anatomy, and functionality that can be used to diagnose, plan, and investigate operations. These images can be either a two-dimensional image or a three-dimensional image, which is solved in terms of both time and non-time.

A large amount of information on these pictures can be evaluated with simple visualization, but for quantitative measurements, the object must be divided into segments. Possible quantitative measurements such as organs size, heart infarct size, muscle weakness, tumor size, as well as tumor

Manuscript received December 5, 2018; revised April 15, 2019.

Sreedhar Kollem is with the JNTUH University, Telangana, India and also Dept of ECE, S R Engineering College (Autonomous), Warangal, India (e-mail: ksreedhar829@gmail.com).

K. Rama Linga Reddy was with G. Narayanamma Institute of Technology and Science, Hyderabad, Telangana, India. He is now with the Department of ETM (e-mail: kattareddy2000@yahoo.com)

D. srinivasa Rao is with the ECE Department, JNTUH College of engineering, Hyderabad, Telangana, India (e-mail: dsraoece@gmail.com). progression or regression or other developmental disorders [1].

Medical imaging analyzes image denoising and segmentation. First, Image denoising [2] stays regular process in digital image processing. It remains a pre-processing period to eradicate certain unidentified such as Additive White Gaussian noise (AWGN) from an image to get a clean image used in favor of image processing, such as image segmentation. Image segmentation is an alternative outlet of the image. A representative instance of the image processor is image denoising prototypical. This method incorporates a disintegrated prototypical giving to some prior information of a corrupted image. To reestablish the distorted image in favor to obtain an auspicious result.

In this instance, $n_{0}$ tells about noisy medical image and $w$ is an original, nevertheless usually known or unknown image i.e.,

$$
n_{0}=D w+n_{1}
$$

in which $D$ is a linear or non-linear operator representing the distortion and $n_{1}$ is additive noise.

Second, segmentation is the most important technique for image processing to identify image objects. Image segmentation stays a progression which splits images into different sections or objects originate on a number of features such as intensity, color or location of the pixels. Segmentation of images is a central theme of analysis about medical image because there are many different types of images and the solution to this problem is different from the application. Some segmentation methods often have poor results, as they do not use previous information about objects containing elements to be segmented [3]. Finally, the image segmentation depends on the image denoising. In recent years, many techniques have been implemented in both image denoising and segmentation. Here is an overview of some techniques, including simple methods that have improved the results in this area. The techniques were explained sequentially in the next section.

The rest of the article is prepared into several sections: i) Literature survey on image denoising and image segmentation methods are offered in Section II; ii) Data availability is introduced in Section III; and iii) Lastly, the conclusion in Section IV is represented.

\section{LITERATURE SURVEY}

\section{A. Image Denoising Methods}

Image denoising is a key issue of image processing applications, such as computer vision and medical science. The performance of the Image denoising method is measured 
by using the important factors such as Mean or Average pixel intensity, Standard Deviation, Mean Square Error, Root Mean Square Error, Mean Absolute Error, Peak Signal to Noise Ratio, Structural Similarity, Universal Image Quality Index, and Entropy [4].

\section{B. Performance Parameters of Image Denoising Methods}

\section{1) Mean}

It determines the dissimilarity of the image by adding all the pixels and dividing the total pixels of the image.

$$
M n=\frac{\sum_{x_{1}=1}^{A} \sum_{x_{2}=1}^{B} Q\left(x_{1}, x_{2}\right)}{A \times B}
$$

where $Q\left(x_{1}, x_{2}\right)$ is the intensity value at the pixel location $\left(x_{1}, x_{2}\right), M n$ is mean or average pixel intensity, $A$ and $B$ are the rows and columns of the image [5].

\section{2) Standard Deviation (SD)}

It refers to the current value of the pixel intensity relative to the image's pixel intensity.

$$
S D=\sigma=\left(\frac{\sum_{x_{1}=1}^{A} \sum_{x_{2}=1}^{B}\left(Q\left(x_{1}, x_{2}\right)-M n\right)^{2}}{A \times B}\right)^{\frac{1}{2}}
$$

\section{3) Average gradient ( $\mathrm{Ag}$ )}

It gives the sharpness and clarity of the image is

$$
A g=\frac{\sum_{x_{1}=1}^{A} \sum_{x_{2}=1}^{B}\left(\left(Q\left(x_{1}, x_{2}\right)-Q\left(x_{1}+1, x_{2}\right)\right)^{2}+\left(Q\left(x_{1}, x_{2}\right)-Q\left(x_{1}, x_{2}+1\right)\right)^{2}\right)^{\frac{1}{2}}}{A \times B}
$$

\section{4) Mean Square Error (MSE)}

It remains to calculate image fidelity or to find the difference between two images respectively.

$$
M S E=\frac{1}{A \times B} \sum_{x_{1}=1}^{A} \sum_{x_{2}=1}^{B}\left[Q\left(x_{1}, x_{2}\right)-\tilde{Q}\left(x_{1}, x_{2}\right)\right]^{2}
$$

where $A$ and $B$ are the rows and columns of the image, $Q$ and $\tilde{Q}$ are the original and enhanced images, $x_{1}$ and $x_{2}$ are integers [6].

\section{5) Root Mean Square Error (RMSE)}

The representation of RMSE is

$$
R M S E=(M S E)^{\frac{1}{2}}=\left(\frac{1}{A \times B} \sum_{x_{1}=1}^{A} \sum_{x_{2}=1}^{B}\left[Q\left(x_{1}, x_{2}\right)-\tilde{Q}\left(x_{1}, x_{2}\right)\right]^{2}\right)^{\frac{1}{2}}
$$

\section{6) Mean Absolute Error (MAE)}

The MAE measurement is accomplished to find proximity between fused and source image. Its mathematical equation is

$$
M A E=\frac{1}{A \times B} \sum_{x_{1}=1}^{A} \sum_{x_{2}=1}^{B}\left|Q_{f}\left(x_{1}, x_{2}\right)-Q_{I}\left(x_{1}, x_{2}\right)\right|
$$

in which $Q_{f}\left(x_{1}, x_{2}\right)$ is a fused image, $Q_{I}\left(x_{1}, x_{2}\right)$ is the source image respectively.

\section{7) Peak Signal to Noise Ratio (PSNR)}

PSNR tells about the revamped image is defined as

$$
\operatorname{PSNR}(d B)=10 \log _{10}\left(\frac{\left(2^{q}-1\right)^{2}}{M S E}\right)
$$

where $q$ gives the number of bits in an image, MSE means mean square error respectively.

\section{8) Structural Similarity (SSIM)}

It tells about the image quality in image processing. SSIM depends on three parameters such as luminance, contrast and structural term. The product of these parameters gives the SSIM of the image. The parameter $L\left(x_{1}, x_{2}\right)$ is the luminance assessment function determines the quality of having only a small margin between two images in terms of mean luminance $\left(\mu_{x_{1}}\right.$ and $\left.\mu_{x_{2}}\right)$. The parameter $C\left(x_{1}, x_{2}\right)$ is the contrast assessment function compute the quality of having only a small margin between two images in terms of standard deviations $\left(\sigma_{x_{1}}\right.$ and $\left.\sigma_{x_{2}}\right)$. The parameter $S\left(x_{1}, x_{2}\right)$ is the structure assessment function determines the correlation coefficient between two images in terms of covariance $\left(\sigma_{x_{1} x_{2}}\right)$.

$$
\operatorname{SSIM}\left(x_{1}, x_{2}\right)=\left\lfloor L\left(x_{1}, x_{2}\right)\right\rfloor a_{1} \times\left\lfloor C\left(x_{1}, x_{2}\right)\right\rfloor b_{1} \times\left\lfloor S\left(x_{1}, x_{2}\right)\right\rfloor c_{1}
$$

where,

$$
\begin{gathered}
L\left(x_{1}, x_{2}\right)=\frac{2 \mu_{x_{1}} \mu_{x_{2}}+U_{1}}{\mu_{x_{1}}^{2}+\mu_{x_{2}}^{2}+U_{1}} \\
C\left(x_{1}, x_{2}\right)=\frac{2 \sigma_{x_{1}} \sigma_{x_{2}}+U_{2}}{\sigma_{x_{1}}^{2}+\sigma_{x_{2}}^{2}+U_{2}} \\
S\left(x_{1}, x_{2}\right)=\frac{\sigma_{x_{1} x_{2}}+U_{3}}{\sigma_{x_{1}} \sigma_{x_{2}}+U_{3}}
\end{gathered}
$$

where $\mu_{x_{1}}$ and $\mu_{x_{2}}$ are the local mean, $\sigma_{x_{1}}$ and $\sigma_{x_{2}}$ are the standard deviation, $\sigma_{x_{1} x_{2}}$ is the cross-covariance of $x_{1}, x_{2}$ for the image. Assume $a_{1}=b_{1}=c_{1}=1, U_{1}=\left(R \times S_{1}\right)^{2}$ and $U_{2}=\left(R \times S_{2}\right)^{2}$.

where $R$ is the size of the image (256 for 8 -bit grayscale images), $U_{1}$ is a small constant value at $S_{1}<<1, U_{2}$ is positive constant value at $S_{2}<<1$ and $U_{3}=U_{2} / 2$.

The simplified version of SSIM is

$$
\operatorname{SSIM}\left(x_{1}, x_{2}\right)=\frac{\left(2 \mu_{x_{1}} \mu_{x_{2}}+U_{1}\right)\left(2 \sigma_{x_{1} x_{2}}+U_{2}\right)}{\left(\mu_{x_{1}}^{2}+\mu_{x_{2}}^{2}+U_{1}\right)\left(\sigma_{x_{1}}^{2}+\sigma_{x_{2}}^{2}+U_{2}\right)}
$$

\section{9) Universal Image Quality Index (UQI)}

It determines image distortion by combining three factors, such as contrast distortion, loss of correlation, and luminance distortion.

$$
U Q I=\frac{\left(4 \times \sigma_{x_{1} x_{2}}\right)\left(\mu_{x_{1}}+\mu_{x_{2}}\right)}{\left(\mu_{x_{1}}^{2}+\mu_{x_{2}}^{2}\right)\left(\sigma_{x_{1}}^{2}+\sigma_{x_{2}}^{2}\right)}
$$




\section{0) Entropy}

Entropy statistical action demonstrates the ailment or intricacy of the image

$$
E=-\sum_{x_{1}=0}^{A-1} \sum_{x_{2}=0}^{B-1} g\left(x_{1}, x_{2}\right) \times \log \left(g\left(x_{1}, x_{2}\right)\right)
$$

where $g\left(x_{1}, x_{2}\right)$ is the intensity value at the pixel location $\left(x_{1}, x_{2}\right), x_{1}$ and $x_{2}$ are integers respectively.

There are other algorithms and general technologies designed intended for image denoising. These are listed below:

\section{Statistical Modeling based image denoising}

In this category, various authors suggest new algorithms to set up statistical models. New algorithms are discussed below.

Wang et al. (2018) [7] demonstrated the non-convex total variation minimization and it is a general technique used in the gradient field as a cross-sectional image. The author discusses the problem of MR brain image reconstruction of extremely subordinate Fourier measurements. The author presents the minimax-concave TV (MCTV) to reconstruct the MR brain image. MCTV is non-convex, mainly to represent the nonconvexity parameter properly and at the same time maintain a convexity for every iteration step. MCTV may receive promotional applications in the area of neurology in the future.

Park et al. (2016) [8] presented parallel imaging using multilayer perception for MR image denoising. The author has several contrasting MR images with similar structural information and noise patterns on images used to realize PI technologies. The author suggested a method of learning-based denoising. This significantly reduces PI noise and the start-up time is much faster. It creates high-quality images that do not have degrading information to increase speed.

Zhang et al. (2016) [9] demonstrated an MRI denoising framework that structurally correlates between users to evaluate the models efficiently to reduce noise in the signal. This eliminates the need for long-term multi-acquisition. This will help the reliability in a survey of the integrity of white material on the development, aging, and disorders.

Waqas et al. (2016) [10] highlighted significant issues in confiscating random noise as of digital images with control filter, in detecting impulse noise, which is a random, shadowed disparity of the image. New noise detection algorithms were proposed based on the grouping of K-means clustering with Non-Local Means based filter. Luo-statistic has been developed a non-local means-based estimator. In this work, the first step in assessing the noise ratio before detecting noise. This estimate agrees to choose appropriate constraints aimed at noise detection procedure. In a faulty phase, pixels are restored to interfere with their actual values. Using this global dataset, this method shows that noise cancellations can be effectively erased. A widespread evaluation of counterfeit results with already reported results reveals that the anticipated process is excessive operative than existing noise abatement technology for both noise and image enhancement.

\section{Derivative-based image denoising}

Magudeeswaran et al. (2017) [11] have launched the concept of a new approach for machine learning and is used to enhance and segment the medical image. The author develops a novel level-set technique originate from the active contour prototypical. This technique diminishes the costly initialization of a level set technique further efficiently. Primarily, images are pre-processed with the Contrast Limited Fuzzy Adaptive Histogram Equalization (CLFAHE) improvement technique to enhance dissimilarity of the image. The improved images remain segmented by means of the projected system. This technique associated the GAC and Chan-Vese $(\mathrm{C}-\mathrm{V})$ prototypical with the advantages of local and global segmentation. In conclusion, image entropy, Absolute Mean Brightness Error (AMBE), PSNR and Feature Similarity Index (FSIM) were found and this gave the best results.

Yang et al. (2016) [12] proposed for image denoising algorithm using PDE and GCV. Through the proposed algorithm, the blocking effect is reduced by tetrolet transformation and is considered to be good. Because suggested procedure used the GCV theory to get finest denoising threshold short of knowledge of the statistical possessions of noise. Related to various comparable denoising procedures, Tetrolet + GCV + PM1 supports PSNR images are best once noise intensity is high. The images taken through T_GCV_PM1 are flat with maintaining the boundaries, facts of the original image.

Sedaaghi et al. (2016) [13] state that diffusion has even been made is an important element of the partial differential equation (PDE) imaging denoising procedures. Generally, diffusion coefficient, Perona-Malik (PM) are extensively cast-off algorithm for noise cancellation based on PDE. Diffusion coefficient PM analyzes the flux. Based on the PM flux analysis aimed at areas with a slope height, the brightness can lead to unwanted ambiguities and conflicts. To solve these problems, the image remains bisected into three slices, depending on gradient level: the area where the slope is inferior to the smoothness of the area, where the slope is between the smoothness and the flux point and the slope area is higher than the flux point. The author defines conditions to remain taken into account in these three parts. Then, all of these terms are calculated after the diffusion is even made. The test results confirm that the proposed method is best by means of signal-to-noise ratio, mean structural similarity, universal quality index, and feature similarity.

Lundervold et al. (2003) [14] confirmed the novel technique aimed at image smoothing about the fourth order PDE technique. This scheme remains monitored in many realistic medical MRI and non-medical synthesized test images are represented with different domains. This algorithm has a good noise reduction without destroying a substantial anatomical or functional detail.

To overcome overshoots and avoid the edge blurring, the author defines the FODE-based edge-conversing regularization filter aimed at noise elimination and is one of non-linear anisotropic diffusion. The comparison with the results clearly shows that the proposed scheme is better than the LLT model about eliminating noise whereas sharply preserving the edge structures by Huang et al. (2011) [15]. 
The author presented piecewise planarity conditions aimed at FO variational denoising methods in the field. Ineffective imitation of FO variational schemes, the author has come up with mathematical approaches for instances variable constraint parameter and stable step size. While PPCs are not fulfilled with the same information, they can be a valuable mathematical tool for creating the ultimate method for PDE-based denoising. The PPC model demonstrated the ability to take measurements measured in PSNR (and visual inspection) better than the PPC model. The FO variational methods can reinstate improved images than other systems, predominantly after embracing the PPCs. FO methods take reinstated texture areas positively, whereas other methods have a tendency on the way to re-establish the boundaries of huge differences evidently by Lim et al. (2009) [16].

To eradicate the staircasing consequence aimed at TV filter together with evading boundaries distorting in support of fourth-order PDE filter, the author suggested the anisotropic diffusion in favor of image denoising. In anticipated prototypical, the $\mathrm{H}-1$-model is treated by way of reliability tenure remains possessed of a TV regularization together with a fourth-order filter. When the pixels are on the boundaries, the TV filter is nominated so that they can retain the edges. If the pixels are part of the flat area, a fourth-order filter is accepted that can remove the staircase artifacts. Hypothetical consequences are seen that the anticipated prototypical exceeds most modern models presented in the form of qualitative and quantitative assessments by Tan et al. (2016) [17].

\section{Image Segmentation Methods}

Image segmentation remains an important issue of image processing applications, such as computer vision and medical science. The segmentation purpose is to split the image into parts, which are a specific application. The quality of image segmentation scheme is estimated according to factors like Similarity Index, Dice Score, Sensitivity or Overlap fraction, Extra Fraction, Specificity, Accuracy.

\section{Performance Parameters of Image Segmentation Methods}

\section{1) Similarity Index (SI)}

SI tells about the mutual value among input MRI brain image and segmented output image.

$$
S I=\frac{2 \times \text { Tru Pos }}{2 \times \text { Tru Pos }+ \text { Fal Pos }+ \text { Fal Neg }}
$$

where Tru Pos is the True Positive, it tells the volume of positive pixels properly segmented. Fal Pos is the False Positive, it gives the volume of positive pixels imperfectly segmented. Fal Neg is the False Negative, it gives the volume of negative pixels incorrectly segmented. Tru Neg is the True Negative, it gives the volume of negative pixels properly segmented.

\section{2) Dice Score (DS)}

It gives comparisons among the segmented section and ground truth section [18].

$$
D S=\frac{2 \times \text { Tru Pos }}{(\text { Tru Pos }+ \text { Fal Neg })+(\text { Fal Pos }+ \text { Tru Pos })}
$$

\section{3) Sensitivity or overlap fraction}

It describes appropriate segmentation associated with input MR brain images. It provided the ability of the test to identify positive results.

$$
\text { Sensitivity }=\frac{\text { Tru Pos }}{(\text { Tru Pos }+ \text { Fal Neg })}
$$

\section{4) Extra Fraction}

Total voxels not detected as a tumor region given by the extra fraction.

$$
\text { Extra Fraction }=\frac{\text { Fal Pos }}{(\text { Tru Pos }+ \text { Fal Neg })}
$$

\section{5) Specificity}

It is based on the ability of the test to identify negative results [19].

$$
\text { Specificity }=\frac{\text { Tru Neg }}{(\text { Tru Neg }+ \text { Fal Pos })}
$$

\section{6) Accuracy}

It is cast-off to estimate the accomplishment of the tumor detection and segmentation system

$$
\text { Accuracy }=\frac{\text { Tru Neg }+ \text { Tru Pos }}{(\text { Tru Neg }+ \text { Tru Pos }+ \text { Fal Neg }+ \text { Fal Pos })}
$$

Various algorithms and techniques are considered to segment the image. These are shown below.

\section{Clustering methods}

Machine learning is an effective way to automatically analyze and diagnose medical images [20]. It can diminish the burden on scientists in the form of radiology, which can study complex or experimental relationships of the empirical data and make the accurate decision [21]. Machine learning algorithms can be planned into different groups based on different ideologies. This methodology is classified into supervised learning, semi-supervised learning, and unsupervised learning algorithms have its origin in the use of model labels of training. During unsupervised learning, we only have one observation and no label information for each sample. In general, these observations or attributes are not caused by perceived or hidden variables. The main objective of unsupervised learning is to explore the relationship between patterns or to find hidden variables behind the observations. The clustering algorithm is a demonstrative method of unsupervised learning. Semi-supervised learning incorporates supervised and unsupervised learning. It uses labeled and unlabelled data during the training process.

In utmost cases, the most commonly used brain tumor segmentation procedures depend on clustering approaches like Fuzzy C-Means, k-mean, Artificial Neural Networks, Vector Machine Support etc. FCM algorithms are discussed in the next section for segmenting brain tumor.

\section{FCM based methods}

FCM remains a clustering technique that splits two or more clusters in the data collection. This scheme [22] is often cast-off in pattern recognition. This procedure works through 
allocating every point allocated to each cluster center through the length amid the cluster and information point. Further details are in the cluster center, where its members are likely to move towards a special cluster center.

The advantages of FCM algorithms are: (1) providing better results for overlapped datasets and better than the rule of k-means. (2) Contrasting the k-means, data points must belong to a single cluster center, sharing a data component with more than one cluster center is provided. As a result, data points can be more than one cluster. (3) the implementation of FCM data into MR data shows the incentive result. Consequently, the FCM for tumor segmentation becomes fertile. In brain tumor segmentation, the brain tumors are divided into tissues, including activated cells, necrotic core, and edema using the unsupervised FCM clustering procedure [23]. With this algorithm, segmentation images can be generated which are the clinically relevant neuro-anatomical and neuropathological tissue contrast media for raw MR images. Then, some researchers learn more about using FCM as functional vectors. MRI images have been processed by a scheme that combines knowledge-based methods through a multispectral histogram study of the action of brain tumor segmentation [24]. According to fuzzy information, a new image segmentation method, for example, the Fuzzy Knowledge-Based Seeded Region Growing [25], is proposed. Investigational outcomes demonstrated that the FKSRG technique has a multi-spectral MR image efficient than automotive segmentation device, k-means, and SVM methods. Because FCM remains an iterative procedure make use of more time. Different solutions, such as Fast Generalized FCM (FGFCM) algorithms and BCSM algorithms, are proposed to reduce the time of implementation of this algorithm. The newly fast and secure FCM framework was assigned for brain tumor segmentation, called FGFCM cluster algorithms, by adding local information [26]. The BCFCM algorithm offers high-quality segmented brain images in actual high-speed ways, it is an outstanding device for virtual brain endoscopy so that segmentation of brain tumor occurs [27].

In order to diminish the sensitivity of the conventional FCM algorithm with Gaussian and impulse noises, a modified FCM-based method was suggested to perform the fast and exact segmentation [28]. The purpose of the method is a scalar feature value in all pixels adjacent to the context-dependent filtering technique, which uses spatial and gray level distances. These functions remain monitored by means of the advanced FCM algorithm histogram-based approach. Towards recover the quality of the FCM algorithm, some investigators introduced a neighborhood attraction that relies on comparative position with the properties of adjacent pixels. Genetic algorithms (GA) are good for finding the best solution, but it is difficult to find a precursor, while particle swarm optimization (PSO) increases the optimum solution. Combining GA and PSO has been presented to determine the optimal value of attractiveness [29]. To improve the precision of tumor stage and size, a combined method of k-means and fuzzy C-mean procedures aimed at the treatment of a tumor segmentation was suggested [30]. This process permits the tumor gain to be segmented with precision and reproduction, which is comparable to the manual segmentation. Additionally, for the segmentation process, it decreases the time.

\section{Traditional methods}

In this article, traditional brain tumor segmentation techniques typically involve typical image processing approaches, like threshold and region-based systems [31]. Two-dimensional image segmentation [32] use threshold-based and region-based approaches.

\section{Threshold-based methods}

The threshold-based approach is modest, efficient method compared to its intensity by one or more thresholds. Current threshold-based approaches remain categories as global and local thresholding. If an image has objects with the same intensity or dissimilarity amid items furthermore high contextual, the global thresholding remains the best option to separate objects and backgrounds. Local threshold values are typically assessed with local statistical properties like average MRI intensity with prior knowledge. In addition, the Gaussian distribution was used to determine the thresholds for common brain MRI images [33].

Due to the specific structure of brain tumors, global and local thresholds are used primarily to regulate the estimated position of brain tumors in the brain. In most cases, the threshold is castoff initial stage in the brain segmentation procedure.

\section{Region-based (RB) methods}

The RB segregation methods scrutinize pixels in the picture furthermore create anonymous regions due to combining zone pixels escorted by characteristics locate over predetermined criterion of similarity [34]. The region growing and watershed segmentation schemes remain part of regional methods are mainly employed in brain tumor segments. The area is an increasingly frequent and widespread regional segmentation method and is used to separate the pixels of the region into an image. Growing areas begin with the unique kernel, which fits the pattern of interest. Neighbors of the kernel remain examined together with the area is accompanied by similar criteria. The range of pixels, intensity pixels, or other image functions decides the similar criteria. Kernels are selected physically or via collecting kernels automatically [35]. The benefit of region growing can divide similar areas and establish connectivity areas.

Some researchers have shown the RG based area remains fulfilled method than NR based approaches with regard to segmentation of the MR images, especially in similar tissues and regions [36].

The main drawback of the RG based approach is the incomplete capacity outcome that restricts the precision of MR image segmentation. Multi-scale watershed transformation methods used by researchers to segment brain tumors [37]. An investigation of watershed segmentation approaches of MRI brain tumor information was achieved. Quantitative and qualitative findings indicate that segmentation time and accuracy have improved considerably and managed segmentation successfully. The investigation also recognized some drawbacks of brain tumor segmentation in the watershed approach. A method has been proposed to improve these disadvantages. A multi-parameter watershed segmentation algorithm used to detect an MRI 2-D and 3-D 
brain tumor [38]. A marker-based better-quality watershed procedure by using the prior information about test images for the brain tumor segmentation was proposed [39]. The watershed segmentation procedure frequently agonizes about over-segmentation. To evade over segmentation and yield a sensible segmentation, some cutting-edge approaches have been projected [40].

The virtuous outcomes of brain tumor segmentation by means of predictable approaches are rigid to achieve. In utmost circumstances, these procedures were considered as a pre-processing period in the brain tumor segmentation. Consequently, more progressive automatic approaches were planned to consensus with the necessities of medical doctors.

\section{Deformable based methods}

Because of the 3-D MRI data presentation, segmentation of the data is difficult [41]. The difficult task is to develop the rudiments of the same construction and participate these rudiments addicted to a coherent and reliable method of structures. Additionally, model-based segmentation approaches with parametric (PDBM) and geometric deformable based methods (GDBM) remain suggested to recover the problem. The normally accepted authority of deformable methods has the capability to segment the image of anatomical structures using the boundaries of image statistics composed through prior information of position, extent, and outline of the constructions. Deformable representations have the opportunity to manage changes in the biological structure. In addition, deformable representations can be used to provide high-quality instinctive mechanisms that enable medical professionals and doctors to acquire the skills needed to perform the interpretive tasks they need.

Various techniques such as PDBM and GDBM are discussed in the subsequent sections.

\section{PDB methods}

Active contour approaches are known as parametric deformable based methods (PDBM) and it is introduced in 1988. Once the initialization has been made, the snake will be able to find the object contour well. In parametric deformable models, the main stage in segmenting the brain tumor is the detection of brain tumor borders. Snakes are widely used for sensing the brain tumor boundary. The reality has shown that identifying the boundary by the snake method is well than traditional edge-detection procedures, such as Laplacian, Canny, and Sobel [42].

Some researchers believe that the external energy of snake function is null at the corners and is positive inhomogeneous regions. Many investigators remain annoying to advance the presentation of snake's process. Gradient Vector Flow (GFF) snake and balloon model remained wished-for to recover brain tumor segmentation on $\mathrm{T}_{1}$ brain tumor [43].

The GVF technique was advised to follow the snake difficulties of the rapid capture range and incapability towards snakes' problems. The balloon prototypical licenses to expand the snake's capture range. Parametric deformation model was projected as a phase of spatial relationships as an improvement to accurately calculate $\mathrm{T}_{1}$ MRI of any type of brain tumor [44]. A method is available that includes the merging of brain scanning of segmentation and deformation of the glioma patients' brain into a standard atlas. The projected technique remains created on the Expectation Maximization (EM) procedure, which includes the glioma development prototypical intended for the atlas, a procedure that replaces the usual atlas with the tumor and edema. The altered atlas remains enumerated in the patient's area in addition to evaluate the posterior probability of the labels of different tissues. EM is an iterative improvement of enumerated constraints, the posterior probability of tissue labels and the constraints of tumor development prototypical. It is noteworthy that sometimes the PDM's initial position requires the manual to be nearly enough to the anticipated limit to evade intersecting inappropriate restrictions.

\section{GDB methods}

Using deformable parametric methods for segmentation of 3-D MRI data, one of the drawbacks is the severity of topological changes managed naturally by splitting and merging of contours. Geometric deformable based methods (GDBM, correspondingly known as level sets) have been wished-for to solve this problem. Even though these methods have greatly amended the initiation of parametric active outlines, and only if the original outline is symmetrically positioned in relation to dominant boundaries, it is difficult to do in practice because many methods of segmenting brain tumors cannot cope with objects formulated regularly [45]. As continuous reproduction snakes have to be carefully initiated and liberated by weak or missing boundary parts, level-set snakes have been offered and have more significant advantages than traditional statistical classification and mathematical morphology. A Charged Fluid Framework (CFF) was presented by some researchers and ruled by Poisson's equation in place of a deformable model to accomplish brain tumor segmentation. Level set formula comprised of a regulatory tenure from which the curve development equation aimed at dynamism reduction was obtained. The consistency of level set functions identified was essentially maintained at the level set regularization tenure in order to confirm a precise calculation, evading costly re-use of the changing fixed level set function.

Some researchers created a local intensity clustering of a brain tumor and other images with the intense disorder have established a local clustering principle function for intensity criteria at each point in the neighborhood. The local clustering principle has been integrated into the Neighbourhood Center to define the energy functionality that has been transformed into a defined level set design. The transmission of dynamism was accomplished in the development of mutually level set progress and a field of bias. A tumor cut procedure was implemented that combines tumor segmentation with cellular automata, and the level set is determined by a tumor map of the probability of introducing spatial smoothness.

In utmost cases, the deformable model is not used alone but is used in conjunction with other procedures such as FCM, MRF, ANN, etc. This may include the advantages of two or more procedures to polish the accuracy of brain tumor segmentation.

\section{Data AvailabiLity}

All brain tumor images are taken from the following 
databases, which are itemized in Table I.

TABLE I: DIFFERENT DATABASES

\begin{tabular}{|l|l|}
\hline Name & Link \\
\hline TumorSim & https://www.nitrc.org/projects/tumorsim \\
\hline 3D Slicer & https://www.slicer.org \\
\hline ISTB & http://www.istb.unibe.ch/research/index_eng.html \\
\hline BrainWeb & http://brainweb.bic.mni.mcgill.ca/brainweb/ \\
\hline
\end{tabular}

\section{CONCLUSION}

This article emphasizes a few non-linear image-denoising methods, even though linear methods remain modest, efficient, and then relaxed to put into action, nevertheless they are limited by high noise density and complex noise patterns. Nonlinear methods were the stage of art denoising approaches. The approaches differ in relation to the category of noise used. All kinds of noise, such as impulse, Gaussian white noise, and speckle, have been studied with techniques related to denoising approaches. Each method in its problematic area has its own performance indicators that may not work and meet requirements in another problem zone. However, complete approaches allied by wavelet domain have achieved excellent results due to their adaptation and noise. But the majority of the approaches gives the better results and appearance can be calculated in terms of Mean or Average pixel intensity, Standard Deviation, Mean Square Error, Root Mean Square Error, Mean Absolute Error, Peak Signal to Noise Ratio, Structural Similarity, Universal Image Quality Index, and Entropy.

This article also delivered an outline of MRI-based brain tumor segmentation approaches.

Different types of present brain tumor segmentation approaches work on the MRI images due to the non-invasive with good tissue contrast of MRI and use classification and clustering approaches, using a variety of features and taking more precisely by region. The persistence of these methods is to make a preliminary decision on the diagnosis, the development of tumor diffusion and treatment planning. Although the majority of tumor segmentation algorithms in the MRI brain have relatively good results in medical image analysis.

Different approaches remain discussed in the paper and each approach has its own unique principle such as precision, validity, and toughness of MRI-based brain tumor segmentation and its associated approaches are previously revealed excessive latent in sensing and investigating tumors in medical training. Nevertheless, the majority of the approaches gives the better results and appearance can be calculated in terms of Similarity Index (SI), Dice Score (DS), Sensitivity or Overlap fraction, Extra Fraction, Specificity, and Accuracy.

\section{ACKNOWLEDGMENT}

The author thanks JNTUH University, G. Narayanamma Institute of Technology and Science, and S R Engineering College (Autonomous).

\section{REFERENCES}

[1] M. Raza, I. Irum, and M. Sharif, "A review of image denoising methods,” Jr Eng Sci Tech Rev, vol. 8, no. 5, pp. 41-48, Dec 2015.
[2] K. R. L. Reddy, D.S. Rao, and K. Sreedhar, "Image denoising by using modified sghp algorithm," Int Jr Electr and Comp Eng, vol. 8, no. 2, pp. 971-978, April 2018.

[3] J. Wang, J. Liu, and Y. Pan, "A survey of MRI-based brain tumor segmentation methods," Tsing Sci and Tech, vol. 19, no. 6, pp. 578-595, Dec 2014.

[4] M. Hussain, S. Rathore, and I. Aksam, "Robust brain MRI denoising and segmentation using enhanced non - local means algorithm," Int Jr Img Syst and Tech, vol. 24, no. 1, pp. 52-66, Mar 2014.

[5] A. Prabha, and S. Saladi, "Analysis of denoising filters on MRI brain images," Int Jr Img Syst and Tech, vol. 27, no. 3, pp. 201-208, Aug. 2017.

[6] V. Kollu and D. P. Bavirisetti, "Fusion of MRI and CT images using guided image filter and image statistics," Int Jr Img Syst and Tech, vol. 27, no. 3, pp. 227-237, Aug. 2017.

[7] Z. Wang, W. Mei, and H. Du, "Convex MR brain image reconstruction via non - convex TV minimization,' Int Jr Img Syst and Tech, vol. 28, no. 4, pp. 1-8, Dec 2018.

[8] H. Park and K. Kwon, "Multi-contrast MR image denoising for parallel imaging using multilayer perceptron," Int Jr Img Syst and Tech, vol. 26, no. 1, pp. 65-75, April 2016.

[9] P. Zhang, and D. Shen, "Denoising MRI using collaborative non-local means," Neu comp, vol. 177, pp. 215-227, Feb 2016.

[10] M. Waqas and S. Alshomrani, "Image de-noising using noise ratio estimation, K-means clustering, and NLM-based estimator," Comp \& Electr Eng, vol. 54, pp. 370-381, Aug 2016.

[11] V. Magudeeswaran and J. Singh, "A machine learning approach for brain image enhancement and segmentation," Int Jr Img Syst and Tech, vol. 27, no. 4, pp. 311-316, Dec 2017.

[12] Y. Yang and Y. Chen, "Image denoising by using PDE and GCV in tetrolet transform domain," Eng Appl Artif Intell, vol. 48, pp. 204-229, Feb 2016.

[13] M. H. Sedaaghi and S. Saryazdi, "Efficient diffusion coefficient for image denoising," Comp \& Math with Appl, vol. 72, no. 4, pp. 893-903, Aug. 2016.

[14] A. Lundervold and X. C. Tai, "Noise removal using the fourth-order PDE with applications to medical MRI in space and time" IEEE Trans on Img Proc, vol. 12, no. 12, pp. 1579-1590, Dec. 2013.

[15] L. Huang and G. Guo, "Adaptive fourth-order PDE filter for image denoising," Appl Math Lett, vol.24, no. 8, pp. 1282-1288, Aug. 2011.

[16] H. Lim, "Fourth-order PDE for effective image denoising," Electr J Diff Equ, vol. 17, pp. 107-121, Jan. 2009.

[17] J. Tan and L. Ai, "Hybrid regularizers-based adaptive anisotropic diffusion for image denoising," SprinPlu, vol. 5, no. 1, p. 404, Dec. 2016.

[18] G. L. Priya, "Walsh Hadamard kernel-based texture feature for multimodal MRI brain tumor segmentation," Int Jr Img Syst and Tech, vol. 28, no. 4, pp. 254-266, Dec. 2018.

[19] A. K. Ray and H. P. Thethi, "Image analysis for MRI based brain tumor detection and feature extraction using biologically inspired BWT and SVM," Int Jr Biomed Img, Mar. 2017.

[20] V. Govindaraj and A. Thiyagarajan, "A fully automated hybrid methodology using Cuckoo-based fuzzy clustering technique for MRI brain image segmentation," Int Jr Img Syst and Tech, vol. 27, no.4, pp. 317-332, Dec. 2017.

[21] N. M. Nasrabadi, "Pattern recognition and machine learning," Jr Electr Img, vol. 16, no. 4, Oct. 2016.

[22] A. Farag and T. Moriarty, "A modified fuzzy c-means algorithm for bias field estimation and segmentation of MRI data," IEEE Trans Med Img, vol. 21, no. 3, pp. 193-199, Mar. 2002

[23] M. S. Silbiger and J. C. Bezdek, "A comparison of neural network and fuzzy clustering techniques in segmenting MRI of the brain," IEEE Trans Neur Net, vol. 3, no. 5, pp. 672-682, Sep. 1992.

[24] M. L. Silbiger, "Application of fuzzy c-means segmentation technique for tissue differentiation in MRI of a hemorrhagic glioblastoma multiforme," Magn Reson Img, vol. 13, no. 2, pp. 277-290, Jan. 1995.

[25] C. C. Kang and C. M. Wang, "Multispectral MRI segmentation based on fuzzy knowledge and modified seeded region growing," Magn Reson Img, vol. 30, no. 2, pp. 230-246, Feb. 2012.

[26] S. M. Szilagyi and H. S. Adam, "MR brain image segmentation using an enhanced fuzzy c-means algorithm," Int Conf IEEE, vol. 1, pp. 724-726, Sep. 2003.

[27] S. Chen and D. Zhang, "Fast and robust fuzzy c-means clustering algorithms incorporating local information for image segmentation," Patt Recog, vol. 40, no. 3, pp. 825-838, Mar. 2007.

[28] S. M. Szilagyi and Z. Benyo, "A modified fuzzy c-means algorithm for MR brain image segmentation," Int Conf Img Anal Recog, pp. 866-877, Aug. 2007. 
[29] N. Forghani and M. Teshnehlab, "Parameter optimization of improved fuzzy c-means clustering algorithm for brain MR image segmentation," Eng Appl Artif Intel, vol. 23, no. 2, pp.160-168, Mar. 2010 .

[30] M. M. Shringirishi, "Implementation of brain tumor segmentation in brain MRI using k-means clustering and fuzzy c-means algorithm," Int Jr Comp Tech, vol. 5, no. 1, pp.54-59, April 2013.

[31] L. Bischof, "Seeded region growing," IEEE Trans Patt Anal Mach Intel, vol.16, no. 6, pp. 641-647, Jun. 1994.

[32] D.C. Gharpure, "Development of image-processing software for automatic segmentation of brain tumors in MRI," Jr Med Phy/Assoc Med Phys of Ind, vol. 36, no. 3.

[33] R. Fahlbusch and O. Ganslandt, "Improved delineation of brain tumors: An automated method for segmentation based on pathologic changes of 1H-MRSI metabolites in gliomas," Neu Img, vol. 23, no. 2, pp. 454-461.

[34] K. P. Wong, "Medical image segmentation: methods and applications in functional imaging," Handbook of Biomed Img Anal, pp. 111-182, 2005.

[35] F. A. Jolesz and R. Kikinis, "Automated segmentation of MRI of brain tumors," Radiogy, vol. 218, no. 2, pp. 586-591, Feb. 2001.

[36] S. M. Alian and M. E. M Bayome, "Validation techniques for quantitative brain tumors measurements," in Proc. Annl Int Conf, 2005 pp. 7048-7051.

[37] D. Bloyet and J. M. Constans, "A framework of fuzzy information fusion for the segmentation of brain tumor tissues on MRI," Img Vis Comp, vol. 25, no. 2, pp. 164-171, Feb. 2007.

[38] S. K. Sharma, "Multiparameter segmentation and quantization of brain tumor from MRI images," Ind Jr Sci Tech, vol. 2, no. 2, pp. 11-15, Feb. 2009.

[39] A. Bahrani, "Segmentation of tumor tissue in gray medical images using the watershed transformation method," Int Jr Advan in Comp Tech, vol. 2, no. 4, Oct. 2010.

[40] Y. Li and B. Zhang, "A novel approach for segmentation of MRI brain images," in Proc. IEEE Mediterranean Electr Conf, 2006, pp. 525-528.

[41] D. L. Arnold and D. L. Collins, "Review of automatic segmentation methods of multiple sclerosis white matter lesions on conventional magnetic resonance imaging," Med img Anal, vol. 17, no. 1, pp. 1-18, Jan. 2013.

[42] J. L. Prince, "Snakes, shapes, and gradient vector flow," IEEE Trans img proc, vol. 7, no. 3, pp. 359-369, Mar 1998.

[43] S. Ourselin, "A new deformable model using dynamic gradient vector flow and adaptive balloon forces," APRS Workshop on Dig Img Comp, pp. 9-14, Feb. 2003.
[44] I. Bloch, "3D brain tumor segmentation in MRI using fuzzy classification, symmetry analysis, and spatially constrained deformable models," Fu Sets Sys, vol. 160, no. 10, May 2009.

[45] D. Valentino, "An electrostatic deformable model for medical image segmentation," Comput Med Img and Grap, vol. 32, no. 1, pp. 22-35 Jan. 2008.

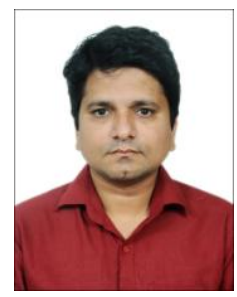

Sreedhar Kollem is a Ph.D. research scholar in the Department of ECE at JNTUH University, Hyderabad, Telangana, India. He received the B.Tech. degree in electronics and communication engineering from JNTUH University, Hyderabad, India in 2005 and $\mathrm{M}$. Tech degree in communication systems from JNTUH University, Hyderabad, India in 2009. He has a life membership in ISTE and IETE. He qualified UGC NET in Electronic Science. His areas of interests are digital image processing, signal processing, and wireless communications.

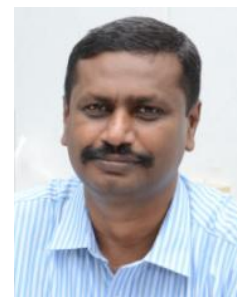

Katta Ramalinga Reddy is working as a professor, head of the Department of Electronics and Telematics (ETM) in G. Narayanamma Institute of Technology and Science (GNITS), Hyderabad, Telangana, India. He completed his B.E. from Vasavi Engineering College in 1989 and M. Tech from SVU Engineering College in 1991, Ph.D. from JNTU Hyderabad. He won the academic excellence award in 2005 for his academic contribution in GNITS. He has more than 40 research papers to his credit. His areas of interests are digital image processing, signal processing, and wireless communications.

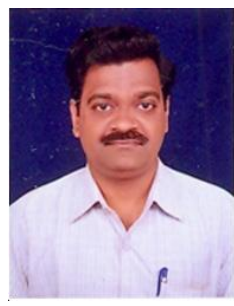

Duggirala Srinivasa Rao is working as a professor of Electronics and Communication Engineering Department at JNTUH College of Engineering, Kukatpally, Hyderabad, Telangana, India. He completed his Ph.D. from Central University Hyderabad. He handled the research projects like Quality of Service in mobile Ad-hoc networks, funded by UGC from 2012 to 2014 . He has more than 35 research papers to his credit. His areas of interests are computer networks, wireless networks, and communications. 